\title{
Maxillofacial Radiology 171
}

SADJ June 2019, Vol. 74 No. 5 p259

\section{CJ Nortjé}

A 40 year old female complains of pain and limitation of movement of her right temporomandibular joint (TMJ). Clinical examination of the joint reveals palpable crepitus and limited movement. What are the important radiological findings and what is your diagnosis?

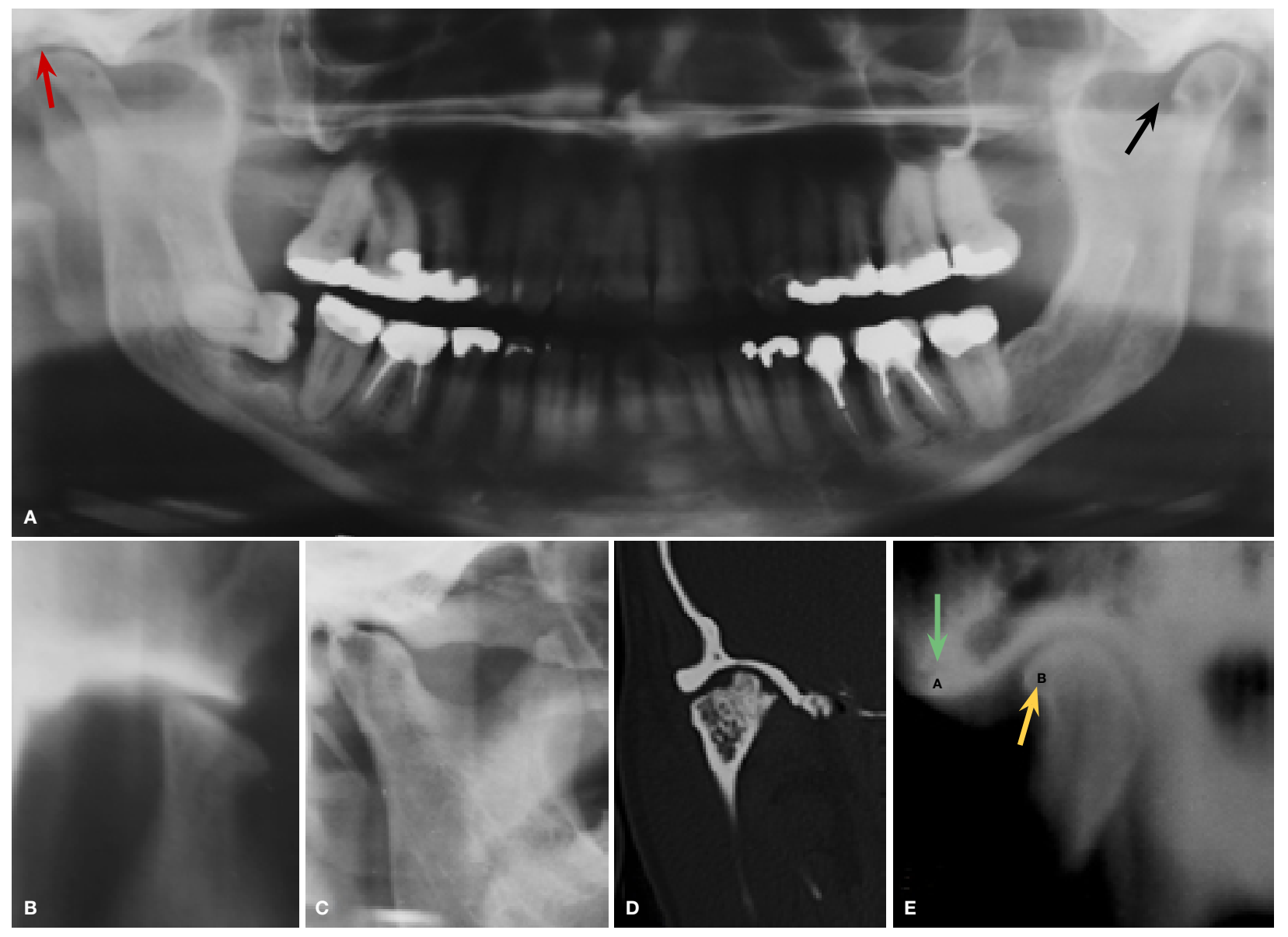

\section{INTERPRETATION}

Symptoms may derive from the impacted and infected 48, osteoarthritis of the TMJ's, joint space narrowed, (red arrow) or an osteophyte (black arrow). Osteoarthritis $(\mathrm{OA})$, the most common type of arthritis (National Institute of Arthritis and Musculoskeletal and Skin Diseases), is caused by normal wear and tear of any joint. The cartilage deteriorates and may even disappear completely, the bone-to-bone contact causing pain, stiffness, and sometimes swelling. OA of the TMJ may include loss of articular cartilage and narrowing of the joint "space" (red arrow), condylar and anterior articular eminence flattening (Fig. B), formation of subcortical cysts and erosion (Fig. C). Axial CT scan (Fig. D) shows OA with a large lateral osteophyte on the superior condylar surface. Conventional tomogram (Fig. E) shows an anterior osteophyte (yellow arrow, dense cortical bone). The green arrow demonstrates eburnation of the eminence (ivorylike reaction of exposed bone). Previously thought

Christoffel J Nortjé: $B C h D, P h D, A B O M R, D S c$. Faculty of Dentistry, University of the Western Cape. Email: cnortje@uwc.ac.za non-inflammatory, osteoarthrosis was, an inadequate misnomer. Now accepted as a low grade inflammatory condition involving all components of the joint, a major cause of pain and disability in adults. About $10 \%$ of men and $18 \%$ of women over 60 years have symptomatic osteoarthritis, commonly of the knee, hip and hand joints, but also the TMJ. Diagnostic imaging (MRI) may be necessary to confirm diagnosis. Disc displacement and osteoarthritis may be shown, since both soft tissue and bone abnormalities can be assessed. However, for bone details CT is generally accepted as superior to MRI. CBCT is increasingly used to assess the TMJ, offering diagnostic accuracy and lower radiation dose, is advisable to ascertain that no other underlying pathology is present.

\section{Reference}

1. Rozylo-Kalinowska, I, Orhan, K: Imaging of the Temporomandibular Joint, Springer Nature Switzerland 2019 p264.

2. Farman AG, Nortje CJ, Wood RE: Oral and Maxillofacial Imaging, $1^{\text {st }}$ Ed, Mosby. St. Louis, Missouri 1993 pp 366-9. 